\title{
CONTRIBUTION A L'ETUDE DU COMPORTEMENT OPTIMUM DE LA CÉDANTE ET DU RÉASSUREUR DANS LE CADRE DE LA THÉORIE COLLECTIVE DU RISQUE
}

\section{H. LAMBERT}

Bruxelles, Belgique

\section{§ I. COUT ET EFFICACITE DE LA REASSURANCE}

a) On peut caractériser la situation du portefeuille d'une société d'assurance par:

le montant de ses réserves libres: $Z$

le coefficient de sécurité attaché aux primes pures $P$ encaissées : $\lambda$ la fonction de fréquence des montants de sinistre: $v(x)$ le bénéfice ou gain moyen par prime pure unitaire: $\delta \lambda$

Aux trois premiers élements correspond une probabilité de ruine $\varepsilon$; nous définissons cette dernière comme la probabilité d'extinction d'un fonds de valeur initiale $Z$, alimenté par des primes $(I+\lambda)$. $\mathrm{P}$ et débité des montants de sinistre $x$ liquidés ou mis en réserve, soit pendant une période infinie ou finie (processus de ruine continu), soit à des époques fixées d'avance, (processus de ruine discontinu).

Cette probabilité de ruine peut, dans des hypothèses assez générales, s'exprimer par:

$$
\varepsilon=C e^{-R Z}
$$

où $C$ est une fonction, dont la valeur est inférieure à l'unité, de $\lambda$ et éventuellement de $Z$ et de la longueur $\mu$ de la période considérée.

$R$, appelé paramètre (ou indice) de sécurité, est solution de l'équation:

$$
\mathrm{I}+(\mathrm{I}+\lambda) R=\int_{0}^{\infty} e^{R x} v(x) d x
$$

dans un processus de ruine continu et non contagieux, si on suppose que $\int_{0}^{\infty} x v(x) d x=\mathrm{I}$ 
$Z$ étant fixé et $\lambda$ étant maintenu constant, il existe donc, après élimination de $R$, une relation entre $v(x)$ et $\varepsilon$ : à différentes formes de $v(x)$ correspondent différentes valeurs de la probabilité de ruine. Or, le but essentiel de la réassurance est de modifier l'expression analytique de la fonction de répartition des montants de sinistres à charge de l'assureur de façon à accroître la sécurité de ses opérations.

Il dispose généralement de deux moyens, employés d'ailleurs simultanément, pour substituer à $v(x)$ une autre fonction $v^{\prime}(x)$ : il peut choisir le mode de réassurance et le niveau $\vee$ de rétention de ses risques.

Par exemple, en réassurance excess loss de plein $n$, les montants de sinistres supportés par la cédante ont une fréquence égale à:

$$
\left\{\begin{array}{cc}
v(x) & \text { pour } x<n \\
\int_{n}^{\infty} v(x) d x & \text { pour } x=n \\
O & \text { pour } x>n
\end{array}\right.
$$

et en réassurance en quote-part (ou en participation) de rapport $\frac{I}{K}\left(^{*}\right)$, la fonction de fréquence est $K v(K x)$.

Par conséquent, dans un mode de réassurance et pour un coefficient de sécurité déterminés, on peut dresser un tableau faisant correspondre, à divers niveaux de rétention $\nu_{i}$, des valeurs différentes $\varepsilon_{i}$ de la probabilité de ruine:

$$
\text { on a } \varepsilon_{i+1}>\varepsilon_{i} \text { si } v_{i+1}>v_{i}
$$

Un tel tableau permet à l'assureur de connaître le risque de ruine qu'il court en adoptant telle ou telle politique de réassurance; cependant, il ne lui donne pas le niveau de rétention qui lui procurera la plus grande sécurité et un gain maximum.

Or, la première question à laquelle nous nous proposons de donner une réponse est la suivante; déterminer les cessions en réassurance (type et rétention) qui procurent a la cédante un accroissement donné de sécurité a un prix minimum.

Pour ce faire nous devons envisager le problème sous un autre

*) $\frac{\mathrm{I}}{\mathrm{K}}$ est la part d'un sinistre conservé par la cédante $(K>\mathrm{I})$ 
angle. $Z$ étant toujours invariable, toute modification de la fonction $v(x)$ entraînera une variation de $\lambda$ si on maintient la probabilité de ruine à son niveau initial $\varepsilon$; par exemple, si le niveau de rétention augmente, le coefficient de sécurité $\lambda$ correspondant à $\varepsilon$ augmentera. Nous appelerons $\lambda_{c}^{\varepsilon}$ le coefficient de sécurité ,nécessaire" à la cédante pour obtenir une probabilité de ruine $\varepsilon$ ou, plus simplement, le coefficient de sécurité correspondant à $\varepsilon$

Nous dirons donc que la valeur optimum du niveau de rétention et le meilleur mode de réassurance sont ceux qui correspondent à un chargement de sécurité ,nécessaire” minimum.

Nous devons cependant encore tenir compte d'un autre élément: les conditions exigées par le réassureur que nous pouvons exprimer par $\lambda_{r}^{*}$, coefficient à appliquer aux primes cédées.

Ainsi, si nous désignons respectivement par $\pi_{c}$ et $\pi_{r}$ la portion de prime pure unitaire conservée par l'assureur et cédée au réassureur, l'expression:

$$
\Delta=\lambda-\lambda_{c}^{\varepsilon} \pi_{c}-\lambda_{r}^{*} \pi_{r}
$$

est différente de zéro et sa valeur dépend du mode de réassurance, du niveau de rétention adoptés et de $\lambda_{r}^{*}$; nous l'appelerons taux d'efficacité.

Dans ces conditions le gain moyen de la cédante passera de $\delta \lambda$ à $\delta \lambda+\Delta$.

Notons ici que $\Delta$ peut être positif ou négatif, mais on peut démontrer qu'il existe toujours une valeur du niveau de rétention telle que $\Delta>0$

Supposons que l'assureur veuille réduire sa probabilité de ruine de $\varepsilon$ à $\varepsilon^{\prime}$, il devra dès lors créditer le fonds de garantie d'un chargement de sécurité supplémentaire $\lambda_{c}^{\varepsilon-\varepsilon^{\prime}} \pi_{c}$ et le taux d'efficacité $\Delta$ sera amputé du même montant et deviendra

$$
\Delta^{\prime}=\Delta-\lambda_{c}^{\varepsilon-\varepsilon^{\prime}} \cdot \pi_{c}
$$

le gain moyen de la cédante sera donc $\delta \lambda+\Delta^{\prime}$ pour une prime pure unitaire; $\Delta^{\prime}$ dépend à la fois de $\lambda_{r}^{*}$, de $\varepsilon-\varepsilon^{\prime}$ et du niveau de rétention.

Il peut être positif ou négatif suivant les valeurs attribuées à ces trois variables. 
La diminution de gain sera donc $-\Delta^{\prime}$ que nous appelerons prix de l'accroissement de sécurité $\varepsilon-\varepsilon^{\prime}$.

Il peut paraître paradoxal que l'on obtienne un prix négatif; en effet, on dit habituellement que l'assureur doit ,payer" pour augmenter sa sécurité financière.

La somme à verser est toujours positive s'il ne réassure pas ses risques; dans ce cas, en effet, il devra prélever sur son profit $\delta \lambda$ le chargement de sécurité nécessaire pour obtenir une probabilité de ruine moins élevée.

Mais par le jeu de la réassurance, nous venons de voir qu'il peut se créer un gain supplémentaire $\Delta(>0)$ - au même titre, par exemple, qu'il y a une réduction de la variance totale des montants de sinistre- et il est possible que le chargement de sécurité supplémentaire $\lambda_{c}^{\varepsilon-\varepsilon} \cdot \pi_{c}$ soit inférieur à ce gain; dans le cas, par exemple, où les conditions du réassureur sont particulièrement favorables et où la différence $\varepsilon-\varepsilon^{\prime}$ n'est pas trop élevée.

L'exemple suivant illustre ce que nous venons d'exposer.

$v(x)=e^{-x} ; Z=$ Ioo; probabilité de ruine: $\varepsilon=$ Io $^{-4} \lambda=0$, Io; $\lambda_{r}^{*}=0, \mathrm{x} 6$

supposons que l'assureur veuille réduire la probabilité de ruine à $\boldsymbol{\varepsilon}^{\prime}=3 \cdot 10^{-8}$ en pratiquant la réassurance excess loss,

Dans ces conditions on a $R \cong$ o, I5

$$
\begin{aligned}
& \mathrm{I}+\lambda_{c}^{\varepsilon}+\lambda_{c}^{\varepsilon-\varepsilon^{\prime}}=\frac{\mathrm{I}-e^{-0,85 n}}{\pi_{c} \cdot 0,85} \\
& \Delta=0, \mathrm{IO}-\lambda_{c}^{\varepsilon} \cdot \pi_{c}-0, \mathrm{I} 6 \pi_{r} \\
& \Delta^{\prime}=0, \mathrm{IO}-\left(\lambda_{c}^{\varepsilon}+\lambda_{c}^{\varepsilon-\varepsilon^{\prime}}\right) \cdot \pi_{c}-0, \mathrm{I} 6 \pi_{r}
\end{aligned}
$$

\begin{tabular}{|c|c|c|c|c|}
\hline$n^{*}$ & $\pi_{c}$ & $\pi_{r}$ & $\Delta$ & $-\Delta^{\prime}$ \\
\hline 0,5 & 0,39347 & 0,60653 & $-0,0053 \mathrm{I}$ & $+0,01089$ \\
\hline $\mathbf{I}$ & 0,63212 & 0,36788 & $+0,001693$ & $+0,00039$ \\
\hline 2 & 0,86466 & o, I 3534 & $+0,02188$ & $+0,01859$ \\
\hline 3 & 0,95021 & 0,04979 & $+0,0140 \mathrm{I}$ & $+0,04233$ \\
\hline 5 & 0,99426 & 0,00574 & $+0,00366$ & $+0,06636$ \\
\hline 10 & 0,99995 & 0,00005 & $+o$, oor & $+0,0763^{\circ}$ \\
\hline$\infty$ & $\mathbf{I}$ & o & o & $+0,0764^{\circ}$ \\
\hline
\end{tabular}

ce qui donne le tableau suivant:

$\left.{ }^{*}\right) n=$ plein excess loss exprimé en fonction du coût moyen d'un sinistre. 
Ces résultats sont loin d'être négligeables si l'on tient compte de ce qu'ils s'expriment en fonction des primes pures totales encaissées.

On voit que le prix de l'accroissement de sécurité fixé représente plus de $7,6 \%$ de l'encaissement s'il n'y a pas de réassurance; si l'assureur réassure ses risques, le prix à payer est inférieur, compte tenu bien entendu du chargement relativement faible demandé par le réassureur; il varie et présente un minimum pour un plein égal au coût moyen d'un sinistre approximativement. Nous verrons plus loin qu'il dépend aussi du mode de réassurance.

Si on fait varier $\varepsilon-\varepsilon^{\prime}$ le graphique suivant permet, à l'assureur, de fixer les meilleures conditions de réassurance qui lui permettent d'obtenir la sécurité désirée.

Prix minimum

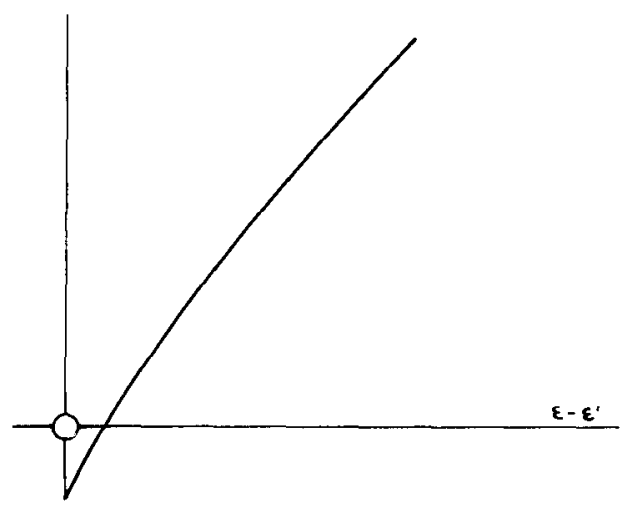

Rappelons que cette courbe rencontre toujours l'axe $\left(\mathrm{O}, \varepsilon-\varepsilon^{\prime}\right)$

b) Nous désignerons par $p(x, v)$ la portion du sinistre de montant $x$, à charge de la cédante dont le niveau de rétention est $v$; la nature de cette fonction dépend du mode de réassurance.

Ainsi en réassurance excess loss

$$
p(x, v)=\left\{\begin{array}{ll}
x & x \leqslant n \\
n & x>n
\end{array} \quad(v \equiv n)\right.
$$

en réassurance de quote-part

$$
\rho(x, v)=\frac{\mathrm{I}}{K} x \quad\left(\frac{\mathrm{I}}{K} \equiv n\right)
$$


$\left.\mathrm{I}^{0}\right)$ Il n'est pas possible de déterminer la forme analytique optimum de $\rho(x, v)$ pour chacun des deux partenaires, cédante et réassureur; les types de cessions habituellement utilisés peuvent néanmoins faire l'objet d'une classification basée sur le taux d'efficacité;

I) On se réfèrera à un article précédent (cfr réf 4) qui montre qu'au point de vue de la cédante, l'efficacité de la réassurance stop loss est supérieure à celle de la réassurance excess loss et que la réassurance en quote-part vient en dernière position (le chargement $\lambda_{r}^{*}$ exigé par le réassureur étant supposé invariable).

2) L'annexe 1 montre que l'ordre de la classification précédente est inversé si on se place au point de vue du réassureur.

Ces résultats ne doivent pas nous surprendre puisque le nivellement parfait des risques n'est obtenu, pour la cédante, que par la réassurance stop loss tandis que la réassurance en participation n'atténue que très imparfaitement leur disparité; d'autre part, ce dernier mode de réassurance protège le réassureur mieux que les autres modes qui lui attribuent la charge intégrale des ,pointes".

Pour employer la terminologie introduite ci-dessus et en vertu de la dualité existant entre la notion d'efficacité et la notion de prix nous dirons que le prix d'un accroissement de sécurité pour la cédante, diminue quand on passe de la réassurance de quote-part à la réassurance excess loss et de cette dernière à la réassurance stop loss.

$\left.2^{0}\right)$ D'autre part, il existe, en excess loss et stop loss, une valeur optimum du plein correspondant à un maximum d'efficacité et par conséquent à un prix minimum.

(cfr réf. 4 et exemple numérique ci-dessus).

Ce maximum est toujours positif quelque grand que soit le chargement de sécurité $\lambda_{r}^{*}$ demandé par le réassureur et l'optimum de $n$, en excess loss, est:

$$
n_{0}=\frac{\mathrm{I}}{R} \lg \left(\mathrm{x}+\lambda_{r}^{*}\right)
$$

On démontre aisément, qu'en réassurance de quote-part il existe également un rapport optimum $\frac{\mathrm{I}}{K_{0}}$ donné par la relation: 


$$
\int_{0}^{\infty} x \cdot e^{-\frac{R}{K_{0}} x} v(x) d x=\mathrm{I}+\lambda_{v}^{*}
$$

Cette solution n'est évidemment acceptable que si $K_{0} \geqslant$ I c'est-àdire si

$$
\mathrm{I}+\lambda_{r}^{*} \leqslant \int_{0}^{\infty} x \cdot e^{R x} v(x) d x
$$

Par conséquent, si le coefficient de sécurité exigé par le réassureur atteint ou dépasse la valeur $\int_{0}^{\infty} x \cdot\left(e^{R x}-\mathrm{I}\right) v(x) d x$ l'assureur n'a aucun intérêt à réassurer ses risques en quote-part.

c) Ces résultats vont nous permettre d'aborder l'étude du comportement de l'assureur envers le réassureur et inversement, dans un mode de réassurance déterminé.

L'intérêt de la cédante $\mathrm{A}$ est d'obtenir du réassureur $X$ les conditions $\left[p(x, v), \lambda_{v}^{*}\right]$ correspondant à un maximum de son taux d'efficacité qui peut s'écrire (cfr annexe 2)

$$
\begin{aligned}
\Delta_{A} & =\frac{\mathrm{I}}{R_{A}} \int_{0}^{\infty}\left[e^{R_{A} x}-e^{R_{A^{P_{A}}}\left(x, \nu_{A}\right)}\right] v_{A}(x) d x- \\
& -\left(\mathrm{I}+\lambda_{r}^{*}\right) \int_{0}^{\infty}\left[x-\rho_{A}\left(x, \nu_{A}\right)\right] v_{A}(x) d x
\end{aligned}
$$

De son côté, le réassureur $X$ s'efforcera de modifier ces conditions à son avantage, c'est-à-dire dans le sens d'un maximum de son taux d'efficacité:

$$
\text { (I.c.2) } \quad \Delta_{X}=\left(\lambda_{r}^{*}-\lambda_{X}\right) \int_{0}^{\infty}\left[x-\rho_{A}\left(x, v_{A}\right)\right] v_{A}(x) d x
$$

ou $\lambda_{x}$ représente le chargement de sécurité „,nécessaire” du réassureur.

Comme nous le verrons plus loin, plusieurs comportements sont possibles; ils diffèrent suivant la structure du marché et le degré d'information que possède l'un des partenaires sur la nature des réactions de l'autre.

Nous pouvons donc déjà affirmer que les intérêts des deux partenaires sont opposés et que leurs négociations aboutiront à se mettre d'accord sur une expression de $p(x, v)$ et une valeur de $v$ et de $\lambda_{r}^{*}$ 
Cependant, les résultats obtenus peuvent très bien ne pas être les meilleurs pour l'ensemble $A+X$; d'une manière plus précise, ces résultats ne conduisent pas nécessairement à un taux d'efficacité globale $\Delta_{A+X}$ maximum.

On observe d'ailleurs que

$$
\begin{aligned}
& \Delta_{A+X}=\frac{\mathrm{I}}{\mathrm{R}_{A}} \int_{0}^{\infty}\left[e^{R_{A} x}-e^{R_{A} \rho_{A}\left(x, y_{A}\right)}\right] v_{A}(x) d x- \\
& -\left(\mathrm{I}+\lambda_{X}\right) \int^{\infty}\left[x-\rho_{A}\left(x, \nu_{A}\right)\right] v_{A}(x) d x
\end{aligned}
$$

qui ne dépend pas de $\lambda_{r}^{*}$ mais varie avec la forme de $\rho$, avec $v$ et $\lambda_{X}$

Nous avons donc affaire à un jeu de deux personnes de somme non nulle.

§2. ETUDE DE QUELQUES COMPORTEMENTS POSSIBLES

a. Si nous reprenons l'expression (I.c.3) nous voyons, de la même manière que pour $\Delta_{A}$, que $\Delta_{A}+X$ possède un maximum pour

$$
n=\frac{\mathrm{I}}{R_{A}} \lg \left(\mathrm{I}+\lambda_{X}\right) \quad \text { en ,excess loss" }
$$

et pour $K_{\mathrm{A}}$ solution de

$$
\int_{0}^{\infty} x e^{\frac{R_{A}}{K_{A}} x} v_{A}(x) d x=\mathrm{I}+\lambda_{X} \text { en ,quote-part" et dans }
$$

chaque cas, ces maxima décroissent quand $\lambda_{X}$ augmente.

D'autre part, l'efficacité de $X$ (cfr I. c.2), négative pour $\lambda_{r}^{*}<\lambda_{X}$ possède un maximum dépendant de la relation qui existe entre $\lambda_{r}$ et $v_{A}$

$\left.I^{0}\right)$ si $\lambda_{r}^{*}$ et $v_{A}$ sont indépendants, $\Delta_{X}$ croît indéfiniment avec $\lambda_{r}^{*}$;

$\left.2^{0}\right)$ si $\lambda_{r}^{*}$ et $\nu_{A}$ sont liés par une relation

$$
v_{A}=f\left(\lambda_{r}^{*}\right)
$$

$\Delta_{X}$ sera maximum pour $\lambda_{r}^{*_{0}}$ solution de 
(2.a.3) $\left(\lambda_{r}^{*_{0}}-\lambda_{X}\right) f^{\prime}\left(\lambda_{r}^{*_{0}}\right)=\frac{\int_{0}^{\infty}\left[x-p_{A}\left(x v_{A}\right)\right] v_{A}(x) d x}{\int_{0}^{\infty} \frac{\partial \rho_{A}}{\delta v_{A}}\left(x, v_{A}\right) v_{A}(x) d x}$

si f est une fonction croissante, ce qui est généralement le cas, on a $\lambda_{r}^{*_{0}}>\lambda_{X}$

Dans ce qui suit, nous supposons que le mode de réassurance est fixé a priori et que les négociations entre $A$ et $X$ portent sur les valeurs de $\nu_{A}$ et de $\lambda_{r}^{*}$ et pas sur la forme de la fonction $p_{A}\left(x, \nu_{A}\right)$

Ier cas

Le marché est déterminé par des conditions $\lambda_{r}^{*}$, identiques pour tous les réassureurs; aucun de ceux-ci ne peut exiger un chargement supérieur à celui du marché et il agirait contre son intérêt s'il demandait un chargement inférieur; d'autre part, l'assureur $A$ agira au mieux de ses intérêts et fixera sa rétention $\nu_{A}$ de façon à maximiser son efficacité. C'est le type de l'adaptation ,ex post" qui implique que $A$ s'adapte à la situation créée par les décisions des réassureurs $X, Y \ldots$ comme si cette situation ne devait pas se modifier à la suite des décisions de $A$.

Dès lors la solution est $\nu_{A}^{0}$ telle que $\frac{d \Delta_{A}}{d \nu_{A}}=0$ d'où $n_{0}=\frac{\mathrm{I}}{R_{A}} \lg \left(\mathrm{r}+\lambda_{r}^{*}\right)$ en ,excess loss" et en "quote-part": a) si $\int_{0}^{\infty} x \cdot e^{R_{A} x} v(x) d x \leqslant \mathrm{I}+\lambda_{r}^{*}$ $K_{0}=$ I, pas de réassurance

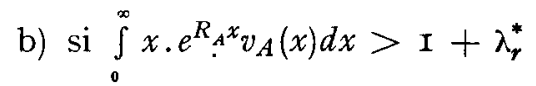
$K_{0}$ solution de $\int_{0}^{\infty} x \cdot e^{\frac{R_{A}}{K_{A}} x} v_{A}(x) d x=\mathrm{I}+\lambda_{r}^{*}$

2ème cas

La stratégie de $X$ est déterminée en fonction des anticipations de la réaction de $A$ aux décisions de $X$; c'est l'adaptation ,,ex ante”. 
$X$ sait donc que $A$ cherchera à maximiser son efficacité et connaît la relation $\nu_{A}=f\left(\lambda_{\eta}^{*}\right)$ que $A$ utilisera pour évaleur l'optimum de $\nu_{A}$

Dans ces conditions, le chargement de sécurité de $X$ sera $\lambda_{r}^{*_{0}}$ solution de:

$$
\frac{\delta \Delta_{X}}{\delta \lambda_{r}^{*}}+\frac{\delta \Delta_{X}}{\delta v_{A}} \cdot f^{\prime}\left(\lambda_{r}^{*}\right)=0
$$

ce qui conduit à l'expression (2.a.3) trouvée ci-dessus et donne: en "excess loss"

$$
\frac{\lambda_{r}^{*_{0}}-\lambda_{X}}{R_{A}\left(\mathrm{I}+\lambda_{r}^{*_{0}}\right)}=\frac{\int_{0}^{\infty} x \cdot v\left(x+n_{0}\right) d x}{\int_{0}^{\infty} v\left(x+n_{0}\right) d x}
$$

$\Delta_{X}$ sera donc maximum, compte tenu de la contrainte $v_{A}=f\left(\lambda_{r}^{*}\right)$, cette fonction étant ici

$$
n_{0}=\frac{\mathrm{I}}{R_{A}} \lg \left(\mathrm{I}+\lambda_{r}^{*_{0}}\right)
$$

et ,en quote-part": $K_{0}^{A}$ et $\lambda_{r}^{*_{0}}$ sont déterminés par les relations

$$
\left\{\begin{array}{c}
\lambda_{r}^{*_{0}}-\lambda_{X}=R_{A}\left(\mathrm{I}-\frac{\mathrm{I}}{K_{0}^{A}}\right) \int_{0}^{\infty} x^{2} \cdot e^{\frac{R_{A}}{K_{0}^{A}} x} \cdot v_{A}(x) d x \\
\int_{0}^{\infty} x \cdot e^{\frac{R_{A}}{K_{0}^{A}} x} \cdot v_{A}(x) d x=\mathrm{I}+\lambda_{r}^{*_{0}}
\end{array}\right.
$$

si celles-ci donnent une valeur $K_{0}$ inférieure à l'unité ou ce qui revient au même si $\lambda_{r}^{*_{0}}>\int_{0}^{\infty} x .\left(e^{R_{A} x}-\mathrm{I}\right) v_{A}(x) d x$ il n'y aura pas de réassurance et les négociations entre $\mathrm{A}$ et $\mathrm{X}$ n'aboutiront pas.

En outre, dans ce cas, si nous supposons que d'autres réassureurs demandent un chargement $\lambda_{y}^{*}$ inférieur à $\lambda_{y}^{*_{0}}, X$ serait forcé de limiter ses conditions à $\lambda_{r}^{*}$ sous peine de voir $A$ s'adresser ailleurs; l'efficacité maximum $\Delta_{X}$ ne serait pas atteinte et nous retombons sur le cas précédent.

D'autre part, nous avons vu que $\lambda_{r}^{*_{0}}>\lambda_{X}$ en général et plus particulièrement en ,excess loss" et "quote-part". 
Par conséquent, le comportement prévu ici ne s'applique que lorsque

$$
\lambda_{X}<\lambda_{r}^{*_{0}} \leqslant \lambda_{r}^{*}
$$

or, nous savons que $\Delta_{A+X}$ est maximum quand $\nu_{A}$ correspond au chargement "nécessaire" $\lambda_{X}$ du réassureur; donc plus on se rapproche de cette dernière valeur, plus l'efficacité globale $\Delta_{A+X}$ augmente. Ce comportement, à ce point de vue, est préférable au premier.

\section{Remarque}

Nous avons supposé, jusqu'à présent, que le partenaire $X$ évaluait avec exactitude les réactions de $A$ et connaissait la fonction $f\left(\lambda_{r}^{*}\right)$; on pourrait envisager le cas où $X$, faute d'information suffisante sur la structure du portefeuille de $A$ (par exemple sur la nature de la fonction $v_{A}(x)$ ) ou sur sa politique (par exemple sur la valeur du paramètre de sécurité $R_{A}$ ) induirait la stratiégie de $A$ suivant un autre critère, au moyen d'une fonction $g\left(\lambda_{r}^{*}\right)$ différente de $f\left(\lambda_{r}^{*}\right)$; il n'y aurait aucune difficulté à déterminer $\lambda_{r}^{*_{0}}$ et $\nu_{A}$ dans cette hypothèse, si la fonction $g\left(\lambda_{r}^{*}\right)$ était connue.

Remarquons aussi, qu'en réassurance excess loss, le montant du plein optimum ne dépend pas de l'expression analytique de la fonction de répartition des montants de sinistre.

\section{zème cas}

Les deux comportements qui précèdent excluent a priori toute collusion entre les deux partenaires, même s'il y a avantage commun, c'est-à-dire même si l'efficacité globale $\Delta_{A+X}$ augmente (comportements concurrentiels).

Maintenant, nous étudierons les conséquences d'un comportement coopératif entre $A$ et $X$ qui déterminent ensemble la meilleure stratégie à suivre pour que l'efficacité globale $\Delta_{A+X}$ soit maxima; cette coalition ne supprime néanmoins pas toute rivalité; cette dernière subsistera dans le partage de $\Delta_{A+X}$.

La solution que Nash (cfr réf. 5) propose est: $\Delta_{A} \Delta_{X}$ maximum; ce qui conduit aux équations: 


$$
\left\{\begin{array}{l}
\Delta_{X} \frac{\delta \Delta_{A}}{\delta \lambda_{r}^{*}}+\Delta_{A} \frac{\delta \Delta_{X}}{\delta \lambda_{r}^{*}}=0 \\
\Delta_{X} \frac{\delta \Delta_{A}}{\delta v_{A}}+\Delta_{A} \frac{\delta \Delta_{X}}{\delta v_{A}}=0
\end{array}\right.
$$

comme $\Delta_{A}$ et $\Delta_{X}$ contiennent, avec des signes opposés, le même terme en $\lambda_{r}^{*}$ la première relation donne $\Delta_{A}=\Delta_{X}$ il y a donc partage pour moitié de l'efficacité globale; cette dernière égalité détermine $\lambda_{r}^{*}$ qui vaut

$$
\lambda_{r}^{*}=\lambda_{X}+\frac{\Delta_{A}}{\int_{0}^{\infty}\left[x-\rho_{A}\left(x, \nu_{A}\right)\right] v_{A}(x) d x}
$$

La seconde relation (2.a.7) est dès lors identique à

$$
\frac{\delta \Delta_{A}+X}{\delta v_{A}}=0
$$

par conséquent, $v_{A}$ doit être tel que l'efficacité globale soit maximum donc en réassurance excess loss, nous aurons:

$$
n_{0}=\frac{\mathrm{I}}{R_{A}} \lg \left(\mathrm{I}+\lambda_{X}\right)
$$

et en réassurance en participation, $K$ sera solution de l'équation

$$
\int_{0}^{\infty} x \cdot e^{\frac{R_{A}}{K} x} \cdot v_{A}(x) d x=\mathrm{I}+\lambda_{X}
$$

et cette solution ne sera acceptable que si

$$
\mathrm{I}+\lambda_{X} \leqslant \int_{0}^{\infty} x \cdot e^{R_{A} x} \cdot v_{A}(x) d x
$$

Remarquons que $\lambda_{y}^{*}>\lambda_{X}, X$ n'a donc aucun intérêt à se retirer de la coalition, bien que son efficacité soit inférieure à celle du 2ème cas. $A$, quant à lui, ne rompra l'accord que si un tiers réassureur $y$ lui offre des conditions telles que son efficacité soit supérieure à $\frac{1}{2} \Delta_{A+X}$.

Il est intéressant de noter que le coefficient de sécurité $\lambda_{X}$ du réassureur décroit quand la puissance financière de ce dernier 
augmente; en effet, plus ses réserves libres sont faibles plus le chargement de sécurité correspondant à une probabilité de ruine déterminée est élevé.

Dès lors, tout arrangement collusoire, en réassurance de quotepart, devient impossible entre une compagnie importante et un réassureur ne disposant pas de moyens financiers comparables.

La réassurance excess loss ne connaît pas cette restriction.

Il existe, en outre, pour l'assureur un avantage, dans ce type de comportement, à choisir un réassureur financièrement puissant, pour deux raisons:

a) l'efficacité globale $\Delta_{A+X}$ et, par conséquent, l'efficacité de $A$, est d'autant plus élevée que $\lambda_{X}$ est faible.

b) le réassureur est en mesure d'offrir des conditions plus avantageuses sans courir de trop grands risques et tout en se réservant une marge bénéficiaire suffisante.

\section{§ 3. LA REASSURANCE RECIPROQUE}

a) Tant que les tractations entre compagnies s'effectuent au sein d'un même portefeuille, il n'est pas nécessaire d'introduire la probabilité $\mu t$ de survenance d'un sinistre.

$\Delta$ représente, dans ce quii précède, l'efficacité pour I unité de prime pure, tandis que l'efficacité pour une unité de coût moyen de sinistre est $\mu . \Delta$.

Si nous considérons deux assureurs $A$ et $B$ dont les densités de probabilité de survenance d'un sinistre sont respectivement $\mu_{A}$ et $\mu_{B}$, l'expression $\left(\mu_{A}+\mu_{B}\right)^{-1} \cdot \mu_{A} \cdot \Delta_{A}$, représente l'efficacité de A pour une unité de prime pure encaissée par l'ensemble $A+B$.

Désignons par $\lambda_{A}, R_{A}, v_{A}(x)$ et par $\lambda_{B}, R_{B}, v_{B}(x)$ les coefficients de sécurité „nécessaires" les paramètres de sécurité et les fonctions de fréquence des montants de sinistres de chacun des portefeuilles $A$ et $B$, respectivement.

L'étude complète des comportements réciproques de deux assureurs, et de leur politique envers un tiers réassureur, nous parait extrêmement complexe: nous donnons toutefois quelques résultats que nous croyons intéressants pour des travaux ultérieurs.

b) Si $A$ et $B$ se réassurent mutuellement leurs risques, l'efficacité globale s'écrit: 


$$
\begin{aligned}
& \text { (3.b.I) } \Delta_{A}+B=\left\{\mu _ { A } \left[\frac{\mathrm{I}}{R_{A}} \int_{0}^{\infty}\left(e^{R_{A} x}-e^{R_{A P_{A}}\left(x, v_{A}\right)}\right) v_{A}(x) d x-\right.\right. \\
& \left.-\left(\mathrm{I}+\lambda_{B}\right) \int_{0}^{\infty}\left[x-\mathrm{\rho}_{A}\right] v_{A}(x) d x\right]+\mu_{B}\left[\frac{\mathrm{I}}{\mathrm{R}_{B}} \int_{0}^{\infty}\left(e^{R_{B} x}-x^{R_{B} \rho_{B}\left(x, v_{B}\right)}\right)\right. \\
& \left.\left.v_{B}(x) d x-\left(\mathrm{I}+\lambda_{A}\right) \int_{0}^{\infty}\left[x-\rho_{B}\right] v_{B}(x) d x\right]\right) \cdot\left(\mu_{A}+\mu_{B}\right)^{-1} \\
& =\left(\mu_{A} \Delta_{A}^{\prime}+\mu_{B} \Delta_{B}^{\prime}\right)\left(\mu_{A}+\mu_{B}\right)^{-1}
\end{aligned}
$$

ou $\Delta_{A}^{\prime}\left(\Delta_{B}^{\prime}\right)$ représente l'efficacité de $A$ (de $B$ ) quand celui-ci cède ses risques à son partenaire qui exigerait un chargement $\lambda_{A}\left(\lambda_{B}\right)$ égal aux coefficients de sécurité ,nécessaires".

En vertu de ce que nous avons vu précédemment $\Delta_{A}$ et $\Delta_{B}$ possèdent chacun un maximum pour des valeurs de $\nu_{A}$ et de $\nu_{B}$ solutions d'une équation du type:

$$
\int_{0}^{\infty}\left(\mathrm{I}+\lambda_{i}-e^{R_{i} \rho_{i}}\right) \frac{\delta \rho_{i}}{\delta v_{i}} v_{i}(x) d x=0 \quad i \equiv A, B
$$

Il en résulte que, en réassurance réciproque, l'efficacité globale n'est pas constante et est une combinaison linéaire de deux efficacités ,marginales" $\Delta_{A}^{\prime}$ et $\Delta_{B}^{\prime}$; de plus, son extrémum correspond aux maxima de ces dernières.

On constate également que le premier terme du zème membre de (3.b.I) représente l'efficacité globale $\Delta_{A+X}$ obtenue si A seulement cédait ses risques à $B$; le second terme étant toujours positif pour $\nu_{B}$ optimum, nous pouvons donc dire qu'au point de vue de l'ensemble $A+B$, la réassurance réciproque est préférable.

D'autre part, si la compagnie $A$ est d'importance beaucoup plus faible que $B$ on aura certainement $\lambda_{A}>\lambda_{B}$; dès lors, le niveau de rétention de $A$ (qui décroît avec $\lambda_{B}$ que ce soit en ,excess loss" ou en "quote-part") sera beaucoup moins élevé que celui de $B$; il y aura donc tendance à ce qu'une grande partie du portefeuille de $A$ soit cédé à $B$, tandis que ce dernier conservera une part relativement grande de ses risques.

c) Précisons la façon dont se feront les échanges entre $A$ et $B$; il semble probable que si deux sociétés s'entendent pour pratiquer la réciprocité, leur comportement mutuel prendra une forme coopérative et, par conséquent, ils chercheront à maximiser l'efficacité globale $\Delta_{A+B}$. 
Cela revicnt à maximiser le produit $\Delta_{A} . \Delta_{B}$ puisqu'ici également $\Delta_{A+B}$ ne dépond pas des chargements de sécurité exigés par $A$ et par $B$.

On aura donc $\frac{\delta}{\delta v_{A}} \Delta_{A+B}=0$ et $\frac{\delta}{\delta v_{B}} \Delta_{A+B}=0$

ce qui revient ̀̀

$$
\frac{\delta}{\delta v_{A}} \Delta_{A}^{\prime}=0 \text { et } \frac{\delta}{\delta v_{B}} \Delta_{B}^{\prime}=0
$$

en vertu de la relation (3.b.I)

Chacun des partenaires maximisera donc son efficacité marginale.

En outre, nous savons que pour une même prime pure nette de réassurance,

Eff. Excess loss $>$ Eff. quote-part d'où

Eff. max. Excess loss > Eff. max. quote-part;

un comportement coopératif conduit donc au choix de la réassurance excess loss de préférence à la réassurance de quote-part comme moyen d'échange des risques de deux assureurs.

Les évaluations numériques suivantes relatives à un cas particulier et donnant les efficacités globales maxima dans chacun des deux modes de réassurance confirment ce résultat.

Efficacité globale maximum $\Delta_{A+B}$

$$
v_{A}(x)=v_{B}(x)=e^{-x} \quad \mu_{A} \equiv \mu_{B}=\mathrm{I}
$$

(Voir le tableau p. 440).

On constate également que l'avantage procuré par la réciprocité est généralement d'autent plus grand que $\lambda_{A}$ ou $\lambda_{B}$ sont élevés.

D'autre part, si $\lambda_{A}^{*}$ et $\lambda_{B}^{*}$ désignent respectivement les chargements exigés par $A$ et par $\mathrm{B}$ sur les cessions du partenaire, les efficacités de $A$ et de $B$ s'écrivent:

$$
\begin{gathered}
\Delta_{A}=\left(\mu_{A}+\mu_{B}\right)^{-1}\left\{\mu_{B} \Delta_{A}^{\prime}+\left(\lambda_{B}-\lambda_{B}^{*}\right) \mu_{A} \int_{0}^{\infty}\left[x-\rho_{A}\left(x, \nu_{A}\right)\right]\right. \\
\left.v_{A}(x) d x+\mu_{B}\left(\lambda_{A}^{*}-\lambda_{A}\right) \int_{0}^{\infty}\left[x-\rho_{B}\right] v_{B}(x) d x\right\} \\
\Delta_{B}=\left(\mu_{A}+\mu_{B}\right)^{-1}\left\{\mu_{B} \Delta_{B}^{\prime}+\left(\lambda_{A}-\lambda_{A}^{*}\right) \mu_{B} \int_{0}^{\infty}\left[x-\rho_{B}\left(x, v_{B}\right)\right]\right. \\
\left.v_{B}(x) d x+\mu_{A}\left(\lambda_{B}^{*}-\lambda_{B}\right) \int_{0}^{\infty}\left[x-\rho_{B}\right] v_{A} d x\right\}
\end{gathered}
$$


440 COMPORTEMENT OPTIMUM DE LA CÉDANTE ET DU RÉASSUREUR

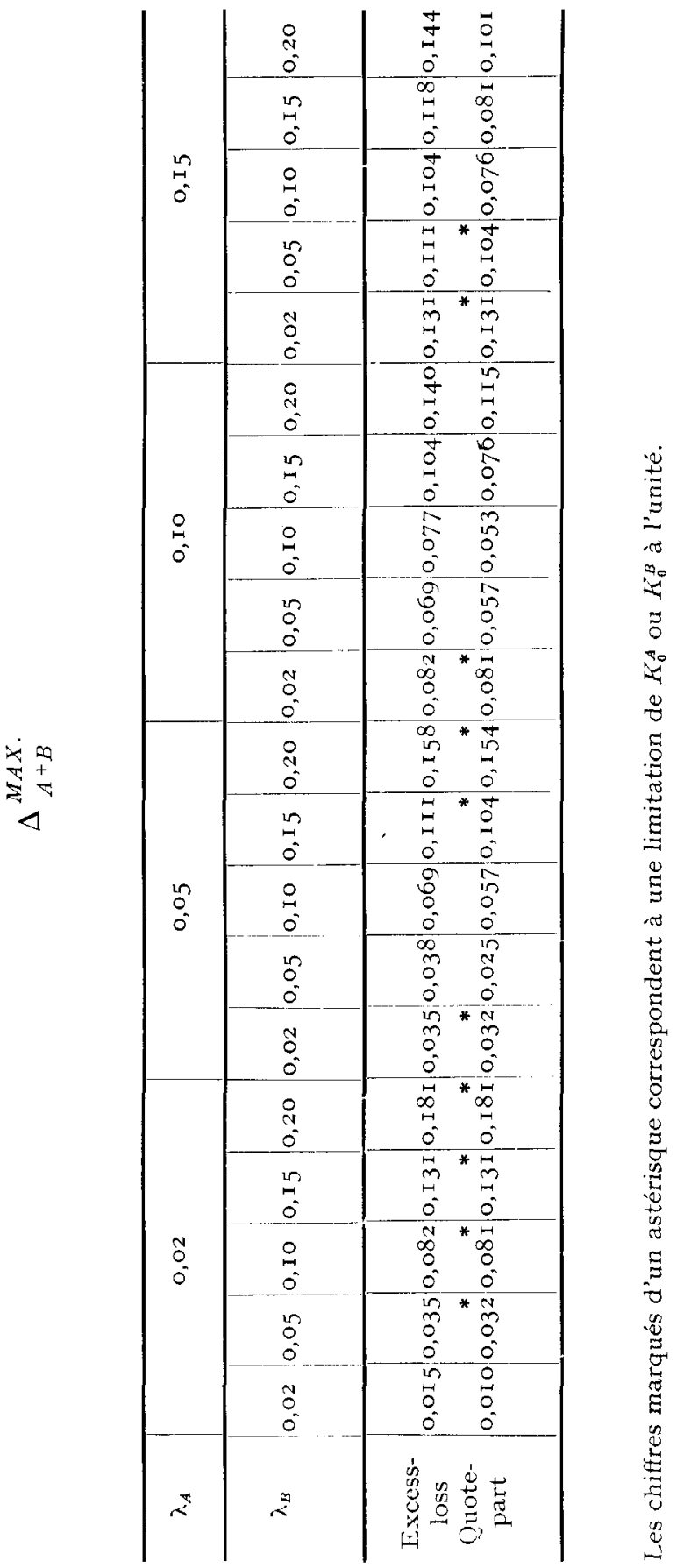


Nous constatons que les termes en $\mu_{A} \lambda_{B}^{*}$ et en $\mu_{B} \lambda_{A}^{*}$ ont les mêmes coefficients et interviennent avec des signes contraires dans les deux expressions ci-dessus; dès lors l'équation

conduit à

$$
\Delta_{A} \frac{\delta \Delta_{B}}{\delta \lambda_{A}^{*}}+\Delta_{B} \frac{\delta \Delta_{A}}{\delta \lambda_{A}^{*}}=0
$$

$$
\Delta_{A}=A_{B}
$$

$\lambda_{A}^{*}$ et $\lambda_{B}^{*}$ devront être tels que l'efficacité globale soit partagée pour moitié entre les deux partenaires.

En outre, en dérivant le produit $\Delta_{A} \Delta_{B}$ successivement par rapport à $\nu_{A}$ et $\nu_{B}$ nous retrouvons la relation (3.c.I):

$$
\frac{\delta \Delta_{A}^{\prime}}{\delta v_{A}}=0 \text { et } \frac{\delta \Delta_{B}^{\prime}}{\delta v_{B}}=0
$$

qui correspondent au maximum de $\Delta_{A}+B$

\section{$\S$ 4. CONCLUSIONS ET CONSIDERATIONS GENERALES}

Nous espérons avoir atteint, en partie du moins, notre but qui était de montrer que les princpes de l'analyse économique peuvent, sans trop de difficultés, être appliqués au problème de la ruine et de mettre en évidence le rôle primordial joué par le chargement de sécurité dans l'élaboration d'une échelle de valeurs pour l'assureur et le réassureur (cfr réf. I).

Si l'un des partenaires fonde sa politique sur d'autres considérations, les résultats de cette étude ne sont naturellement pas valables.

Ainsi, les objectifs poursuivis par certaines compagnies peuvent se circonscrire à l'octroi, par leur réassureur, d'une aide financière ou technique; ce dernier est donc réduit au rôle de prêteur ou de conseiller; de telles considérations, qui sont étrangères à la recherche d'une plus grande sécurité, sortent du cadre de cette étude. Celle-ci ne s'applique également pas au cas où l'assureur assimile la ruine à la survenance d'un solde déficitaire à la fin d'un seul exercice; on conçoit d'ailleurs mal qu'une entreprise élabore sa politique de réassurance en poursuivant des objectifs à si court terme et en faisant abstraction du caractère permanent de ses opérations.

D'autres sociétés, par contre, se préoccupent d'éliminer le plus possible, les fluctuations dans les résultats financiers; ce problème, 
qui nécessite la création d'une réserve de garantie, trouve son application ici.

Les développements qui précèdent ne s'appliquent qu'aux processus de ruine pour lesquels la relation (I.a.I) a la forme indiquée; il est toutefois évident que les notions d'efficacité et de prix sont également valables dans des processus contagieux et discontinus; seules leur forme analytique et les conditions d'extrémum se modifieront. En outre, un comportement coopératif conduira toujours à une efficacité globale maximum et son partage entre les partenaires se fera toujours suivant les mêmes critères (cfr réf. 3)

D'autre part, nous avons supposé que chaque partenaire agit d'une manière rationelle, c'est-à-dire que non seulement il possède, mais il emploie les moyens appropriés à la fin poursuivie; il est supposé pouvoir, par exemple, décider si telle solution est plus avantageuse qu'une autre et prendre toutes les initiatives nécessaires pour la réaliser; son comportement est donc conforme à l'axiome de transitivité. En outre, il est évident que le taux d'efficacité satisfait à l'axiome de continuité.

En résumé, nous devons reconnaître que chacun des partenaires, dans un marché, procède selon l'idiosyncrasie qui lui est propre en appliquant la stratégie dont il est capable ou qu'il croit efficace; ce n'est pas la valeur réelle de ia stratégie qui entre en jeu mais sa valeur psychologique.

Ces quelques réflexions et le fait que les types de comportements étudiés sont schématiques restreignent sensiblement la portée pratique immédiate de certains résultats et ne permettent pas d'entrevoir l'élaboration d'une théorie générale de la réassurance.

Les difficultés que rencontre toute théorie économique dans la représentation exacte du comportement des individus, ne sont-elles pas du même ordre?

\section{BIBLIOGRAPHIE SOMMAIRE}

I) K. Borch: Elements of a Theory of Reinsurance (The Journal of Insurance; vol. XXVIII $\mathrm{N}^{\circ} 3$ )

2) R. Denem: Traité d'Analyse économique.

3) H. Lambert: Quelques aspects du problème de la ruine (Bulletin A.A.Br. 1960)

4) H. Lambert: Une application de la théorie collective du risque: la réassurance (Bulletin A.R.A.B. I960)

5) R. NasH: The Bargaining Problem (Econometrica vol. I 8-1950) 


\section{ANNEXE I.}

a) La réassurance excess loss est, pour le réassureur, moins efficace que la réassurance en quote-part pour une même prime pure cédée.

Il suffit de démontrer que

$$
\lambda_{X}^{(E L)}>\lambda^{(Q P)}
$$

ces notations désignant les chargements de sécurité ,nécessaires” au réassureur $X$, respectivement en ,excess loss” et en , quote-part”.

Or, d'une façon générale, on a

$$
\lambda_{X} \int_{0}^{\infty}[x-\rho(x, v)] v(x) d x=\frac{\mathrm{I}}{R_{X}} \int_{0}^{\infty}\left\{e^{R_{X}[x-\rho]}-(x-\rho) R_{X}-\mathrm{I}\right\} v(x) d x
$$

il revient donc à démontrer que l'expression

$$
\int_{0}^{\infty} e^{R_{X}[x-\rho]} v(x) d x
$$

est plus élevée en réassurance excess loss, les autres termes de la dernière égalité étant constants.

Or, on voit aisément que:

$$
\int_{0}^{\infty} e^{R x} d v(x+n)>\int_{0}^{\infty} e^{R x} d v\left(\frac{K}{K-\mathrm{I}} x\right)
$$

si

$$
\int_{0}^{\infty} x d v(x+n)=\int_{0}^{\infty} x d v\left(\frac{K}{K-I} x\right)
$$

(égalité des primes pures)

$$
\text { c.q.f.d. }
$$

b) La réassurance stop loss est, pour le réassureur, moins efficace que la réassurance excess loss, pour un même montant total annuel de primes pures.

$\mathrm{Si}: G_{n}(x)$ représente la fonction de distribution des montants annuels totaux de sinistre à charge du réassureur qui accepte les risques en ,excess loss" de plein $n$.

$F(x)$ est la fonction de distribution des montants annuels totaux des sinistres (part de la cédante + part du réassureur) les coefficients 
444 COMPORTEMENT OPTIMUM DE LA CÉDANTE ET DU RÉASSUREUR de sécurité ,nécessaires” au réassureur sont, en ,excess loss” et en ,stop loss", donnés respectivement par:

$$
e^{\left(1+\lambda_{x}^{E L}\right) u R_{X}}=\int_{0}^{\infty} e^{R_{X} x} d G_{n}(x)
$$

et

$$
e^{\left(1+\lambda_{X}^{S}\right) u R_{X}}=\int_{0}^{\infty} e^{R_{X}(x-N)} d F(x)
$$

$N$ étant le plein stop loss.

$$
\text { Supposant que } \int_{N}^{\infty}(x-N) d F(x)=\int_{0}^{\infty} x d G_{n}(x)
$$

nous devons prouver que

$$
\lambda_{x}^{(E L)}<\lambda_{x}^{(S)}
$$

ou si on veut:

$$
\int_{0}^{\infty} e^{R_{X} x} d G_{n}(x)<\int_{0}^{\infty} e^{R_{X} x} d F(x+N)
$$

démonstration qui se fait facilement à l'aide d'un graphique, comme la précédente.

ANNEXE 2.

On a:

$$
\Delta_{A}=\lambda-\lambda_{c}^{\varepsilon} \cdot \pi_{c}-\lambda_{r}^{*} \cdot \pi_{r}
$$

avec

$$
\mathrm{I}+\left(\mathrm{I}+\lambda_{c}^{\varepsilon}\right) R_{A} \pi_{C}=\int_{0}^{\infty} e^{R_{A} \rho_{A}\left\langle x, v_{A}\right\rangle} v_{A}(x) d x
$$

en soustrayant cette dernière relation de la relation de Lundberg (I.a.I) en obtient:

$$
\lambda-\lambda_{c}^{\varepsilon} \cdot \pi_{c}+\pi_{r}=\frac{\mathrm{I}}{R_{A}} \int_{0}^{\infty}\left[e^{R_{A} x}-e^{R_{A} P_{A}\left(x, \nu_{A}\right)}\right] v_{A}(x) d x
$$

dont découle directement la relation (I.c.I) 